\title{
MRI histogram analysis enables objective and continuous classification of intervertebral disc degeneration
}

\author{
Christian Waldenberg $^{1,2}$ (1) Hanna Hebelka ${ }^{3,2} \cdot$ Helena Brisby ${ }^{4,2} \cdot$ Kerstin Magdalena Lagerstrand $^{1,2}$
}

Received: 2 January 2017/Revised: 8 August 2017/Accepted: 11 August 2017/Published online: 18 August 2017

(C) The Author(s) 2017. This article is an open access publication

\begin{abstract}
Purpose Magnetic resonance imaging (MRI) is the best diagnostic imaging method for low back pain. However, the technique is currently not utilized in its full capacity, often failing to depict painful intervertebral discs (IVDs), potentially due to the rough degeneration classification system used clinically today. MR image histograms, which reflect the IVD heterogeneity, may offer sensitive imaging biomarkers for IVD degeneration classification. This study investigates the feasibility of using histogram analysis as means of objective and continuous grading of IVD degeneration.

Methods Forty-nine IVDs in ten low back pain patients (six males, 25-69 years) were examined with MRI (T2weighted images and T2-maps). Each IVD was semi-automatically segmented on three mid-sagittal slices. Histogram features of the IVD were extracted from the defined regions of interest and correlated to Pfirrmann grade.

Results Both T2-weighted images and T2-maps displayed similar histogram features. Histograms of well-hydrated IVDs displayed two separate peaks, representing annulus fibrosus and nucleus pulposus. Degenerated IVDs displayed decreased peak separation, where the separation
\end{abstract}

Christian Waldenberg

christian.waldenberg@vgregion.se

1 Department of Medical Physics and Techniques, Sahlgrenska University Hospital, Gothenburg, Sweden

2 Institute of Clinical Sciences, Sahlgrenska Academy, University of Gothenburg, Gothenburg, Sweden

3 Department of Radiology, Sahlgrenska University Hospital, Gothenburg, Sweden

4 Department of Orthopaedics, Sahlgrenska University Hospital, Gothenburg, Sweden was shown to correlate strongly with Pfirrmann grade $(P<0.05)$. In addition, some degenerated IVDs within the same Pfirrmann grade displayed diametrically different histogram appearances.

Conclusions Histogram features correlated well with IVD degeneration, suggesting that IVD histogram analysis is a suitable tool for objective and continuous IVD degeneration classification. As histogram analysis revealed IVD heterogeneity, it may be a clinical tool for characterization of regional IVD degeneration effects. To elucidate the usefulness of histogram analysis in patient management, IVD histogram features between asymptomatic and symptomatic individuals needs to be compared.

Keywords Histogram analysis - IVD classification · MRI · Heterogeneity · Diagnosis

\section{Introduction}

The most frequent source of chronic disability for both genders in working years is low back pain (LBP) [1, 2] resulting in major consequences for both the individual as well as for the society. The associated direct and indirect annual total costs for back pain are estimated to $1-2 \%$ of GDP [3, 4]. Degeneration of intervertebral discs (IVDs) is strongly associated with LBP and suggested to be the most common cause of LBP [5-7].

Magnetic resonance imaging (MRI) is sensitive to changes in cartilage structure and considered to be the best imaging method to evaluate IVD degeneration [8]. However, as conventional IVD degeneration measurements are qualitative and based on visual interpretation of MR images [9], the technique is currently not utilized in its full capacity. Qualitative grading of IVD degeneration is 
confined by subjective interpretation and limit degeneration classification to non-continuous grades. MRI sequences, enabling direct quantification of $\mathrm{T} 1 \rho$ and $\mathrm{T} 2$ relaxation time, have been developed and have showed promise for tissue characterization of the IVD [10-12].

As MR images display regional variations in signal intensity, i.e., pixel values, across the IVD tissue, the technique allows for quantification of the IVD heterogeneity. The signal variation in the MR images examined with histogram analysis may produce histogram features reflecting (1) the regional composition of the IVD tissue and (2) the grade of IVD degeneration [13], none of which is always visually apparent in MR images. If such histogram features would vary between LBP patients and individuals without pain, IVD histogram analysis may also be an important tool to identify painful IVDs.

The aim of this study was to examine the feasibility of histogram analysis to decode IVD heterogeneity with purpose not only to obtain a tool for objective and continuous grading of IVD degeneration but also to generate an indirect detailed map of how the regional IVD structures are affected by degeneration.

\section{Materials and methods}

\section{Patients/intervertebral discs}

To obtain a tool for objective and continuous grading of IVD degeneration, ten patients with chronic LBP (minimum 6 months) with different grade of IVD degeneration (total of 49 IVDs, 6 males, 25-69 years, mean 41 years, median 36 years) were examined on a Siemens Magnetom Aera 1.5 T MRI scanner (Erlangen, Germany). All patients had "non-specific" LBP, i.e., patients with apparent LBP causes, other than degenerated disc disease, were excluded. One IVD was excluded from the analysis due to the IVD being rudimentary. See Table 1 for the distribution of IVD degeneration among the IVDs. All discs were visually evaluated regarding degeneration grade, using Pfirrmann classification, by an experienced radiologist [9].

\section{Magnetic resonance imaging protocol}

For morphological IVD imaging, high quality standardized sagittal T1-weighted (T1W) MRI was performed $[320 \times 320$ matrix, slice thickness $3.5 \mathrm{~mm}$, field of view (FOV) $300 \times 300 \mathrm{~mm}^{2}$, number of excitations (NEX): 2]. Two MRI sequences, both sensitive to the water and proteoglycan content in the IVD tissue and the orientation and concentration of collagen fibers $[14,15]$, were also used reflecting the functional information of the IVD tissue. Namely, T2W-imaging which generate qualitative data
$(384 \times 384$ matrix, slice thickness $3.5 \mathrm{~mm}$, FOV $300 \times 300 \mathrm{~mm}^{2}$, NEX: 1) and T2-mapping generating quantitative data $(256 \times 256$ matrix, slice thickness $3.5 \mathrm{~mm}$, FOV $220 \times 220 \mathrm{~mm}^{2}$, NEX: 1$)$.

\section{Post processing}

Post processing of the image data was performed using MATLAB software R2016a (Mathworks ${ }^{\circledR}$, Natick, Massachusetts, USA). Each IVD was outlined on three consecutive mid-sagittal morphological T1W-images using semi-automated segmentation based on a combination of a region growing algorithm and manual adjustment (Fig. 1). The segmented "regions of interest" (ROIs) were then transferred and rescaled to match the corresponding T2Wimage and T2-map, generating grey scale distributions consisting of two major sections; high and low signal values. The separation between the sections was determined from the peak separation of a two component Gaussian probability distribution model estimating the grey scale distribution. The reproducibility error of the peak separation was estimated by repeating the modelling 1000 times per IVD and was found to be small for all Pfirrmann grades $1-4: \mathrm{CV}=0.1,0.8,3.6$ and $3.6 \%$. The Gaussian peak separation $(\Delta \mu)$ was plotted as a function of Pfirrmann grade for both T2W-images and T2-maps. Furthermore, the mean IVD signal intensity (mean SI) and the mean IVD T2 relaxation time (mean T2) were plotted against Pfirrmann grade. For comparison, $\Delta \mu$ was also plotted against mean SI and mean T2.

\section{Statistical analysis}

Mann-Whitney $U$ test was performed to examine statistical significance between histogram features and Pfirmann grade. Additionally, the correlation between $\Delta \mu$ and mean T2, as well as $\Delta \mu$ and the mean SI, was tested for significance. Statistical significance was defined as $P<0.05$. All values are given as mean \pm one standard deviation, if not otherwise stated.

\section{Results}

\section{Histogram distribution}

For well-hydrated IVDs, the histogram distribution displayed two well separated peaks; one from the low signal intensity in annulus fibrosus (AF), and one from the high signal intensity in nucleus pulposus (NP). Histograms of degenerated IVDs displayed reduced distance between the two peaks, reflecting reduced distinction between $\mathrm{AF}$ and NP. This was displayed by increased number of pixels with 
Table 1 Histogram features and its values for IVDs of Pfirrmann grade 1-4

\begin{tabular}{|c|c|c|c|c|c|}
\hline & & \multicolumn{4}{|c|}{ Pfirrmann grade (Pf) and Group size (n) } \\
\hline & & $P f=1, n=3$ & $\mathrm{Pf}=2, \mathrm{n}=24$ & $\mathrm{Pf}=3, \mathrm{n}=12$ & $\mathrm{Pf}=4, \mathrm{n}=10$ \\
\hline$\Delta \mu$ (pixel intensity) & $\mathrm{B}$ & $161 \pm 29$ & $141 \pm 20$ & $74 \pm 29$ & $43 \pm 12$ \\
\hline$\Delta \mu[\mathrm{ms}]$ & $\mathbb{Z}$ & $116 \pm 7$ & $83 \pm 19$ & $38 \pm 15$ & $24 \pm 8$ \\
\hline Mean pixel intensity & $\mathbf{B}$ & $193 \pm 11$ & $154 \pm 23$ & $96 \pm 19$ & $63 \pm 10$ \\
\hline \multirow[t]{6}{*}{ Mean T2 [ms] } & 8 & $117 \pm 13$ & $89 \pm 18$ & $52 \pm 10$ & $38 \pm 6$ \\
\hline & & & \multicolumn{3}{|c|}{ L } \\
\hline & & & \multirow{2}{*}{$\begin{array}{l}\mathrm{P}=0.000^{*} \\
\mathbf{P}=0.000^{*}\end{array}$} & $00 * \quad \mathrm{P}$ & $04^{*} \mathbb{Z} \mathrm{P}=0.011^{*}$ \\
\hline & & & & DP & $00^{*} \quad \mathrm{P}=0.001$ * \\
\hline & & & & $000^{*}$ & $0 *$ \\
\hline & & & & $.000 * \mathrm{P}$ & $0 *$ \\
\hline
\end{tabular}

Pfirrmann group 1 was not tested for significance due to small group size $(n=3)$. * indicate significance

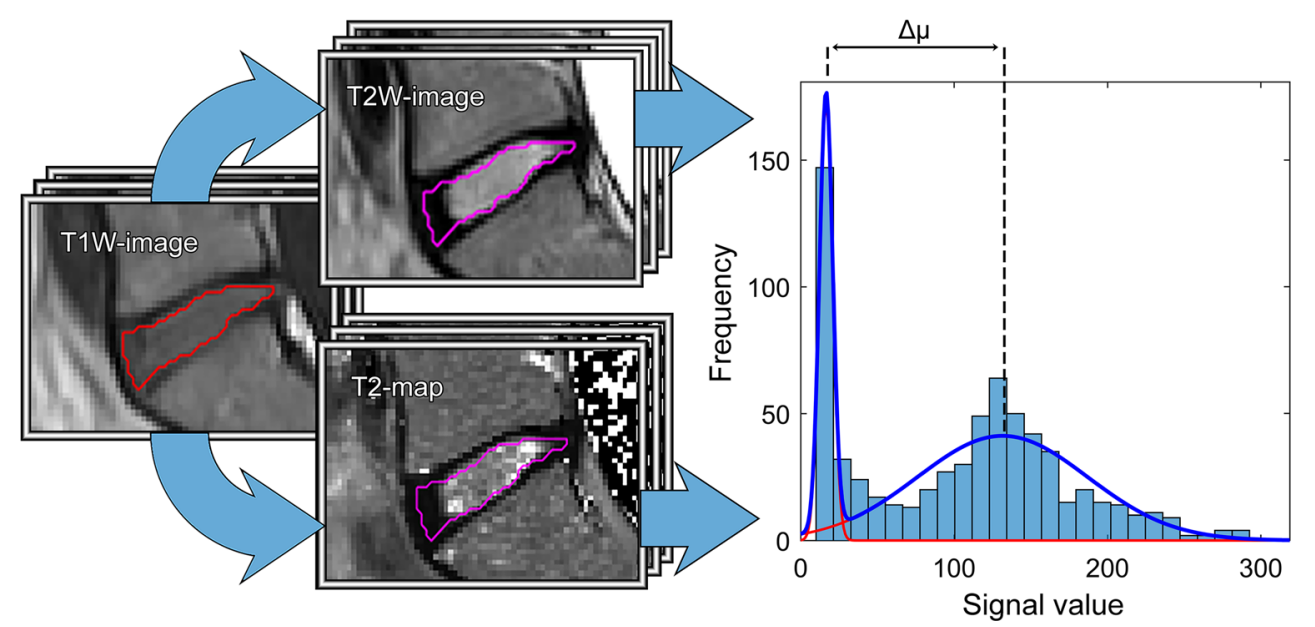

Fig. 1 Schematic illustration of the workflow for retrieving histogram features. ROIs were placed on three mid-sagittal T1W-images which subsequently were transferred and rescaled to match $\mathrm{T} 2 \mathrm{~W}$ -

intermediate grey scale values and decreased peak separation (Fig. 2).

\section{Correlation between histogram features and Pfirrmann grade}

Both the T2W-images and T2-maps displayed similar histogram features (Fig. 3). The peak separation of the Gaussian distributions $(\Delta \mu)$ was shown to correlate strongly with Pfirrmann grade $(P<0.02$; Table 1$)$ where a decreased separation was observed with increased Pfirrmann grade. Similarly, the mean IVD signal intensity (mean SI) and mean IVD T2 relaxation time (mean T2) images and T2-maps. The separation between high and low grey scale values, $\Delta \mu$, was retrieved from analysis of histograms reflecting image data

decreased with increased Pfirrmann grade $(P<0.01)$. Besides this general behaviour, some IVDs displayed histogram topology that notably deviated from the rest of the group (Fig. 4).

\section{Correlation between different histogram features}

$\Delta \mu$ correlated linearly with mean SI in the T2W images ( $R=0.90, P<0.01$ ) and mean T2 in the T2-maps (Fig. 5: $R=0.97, P<0.01)$. As it has previously been shown that T2 correlate with IVD degeneration [14, 16, 17], these findings further emphasize the correlation between $\Delta \mu$ and IVD degeneration. 

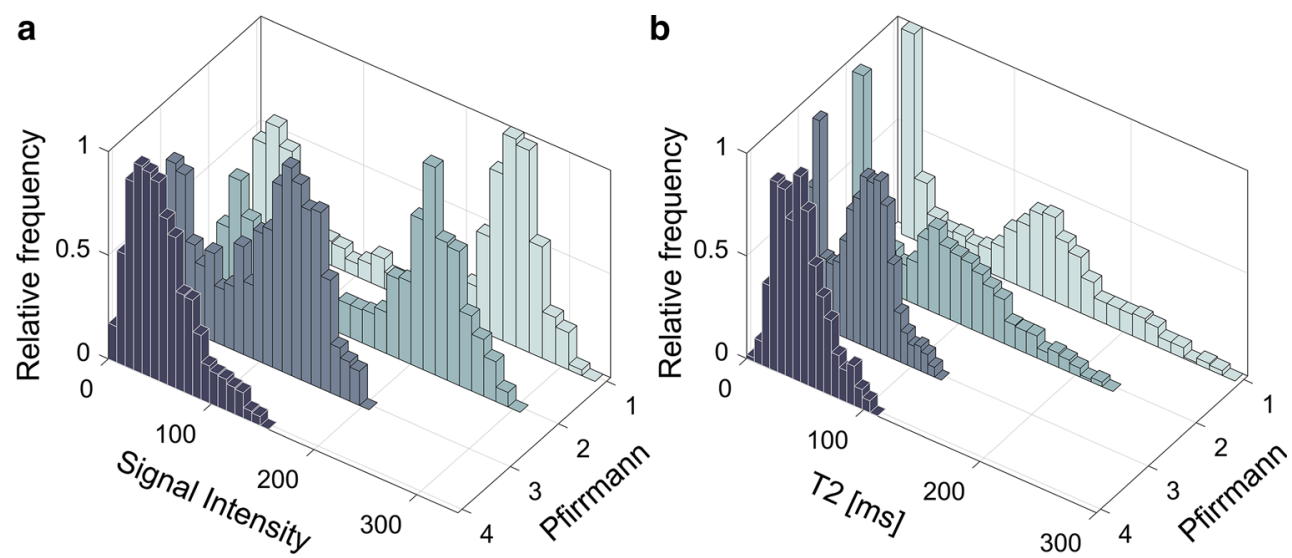

Fig. 2 Typical histograms for a T2W-images and b T2-maps of discs with different grade of degeneration measured in terms of Pfirrmann grading

Fig. 3 Pfirrmann grade versus a $\Delta \mu$ based on $\mathrm{T} 2 \mathrm{~W}$-images, b $\Delta \mu$ based on T2-maps, $\mathbf{c}$ mean disc pixel intensity based on T2W-images and $\mathbf{d}$ mean disc T2 relaxation time based on $\mathrm{T} 2$ maps. The length of a whisker represents \pm one standard deviation from the mean denoted in parenthesis. Each marker represents one intervertebral disc
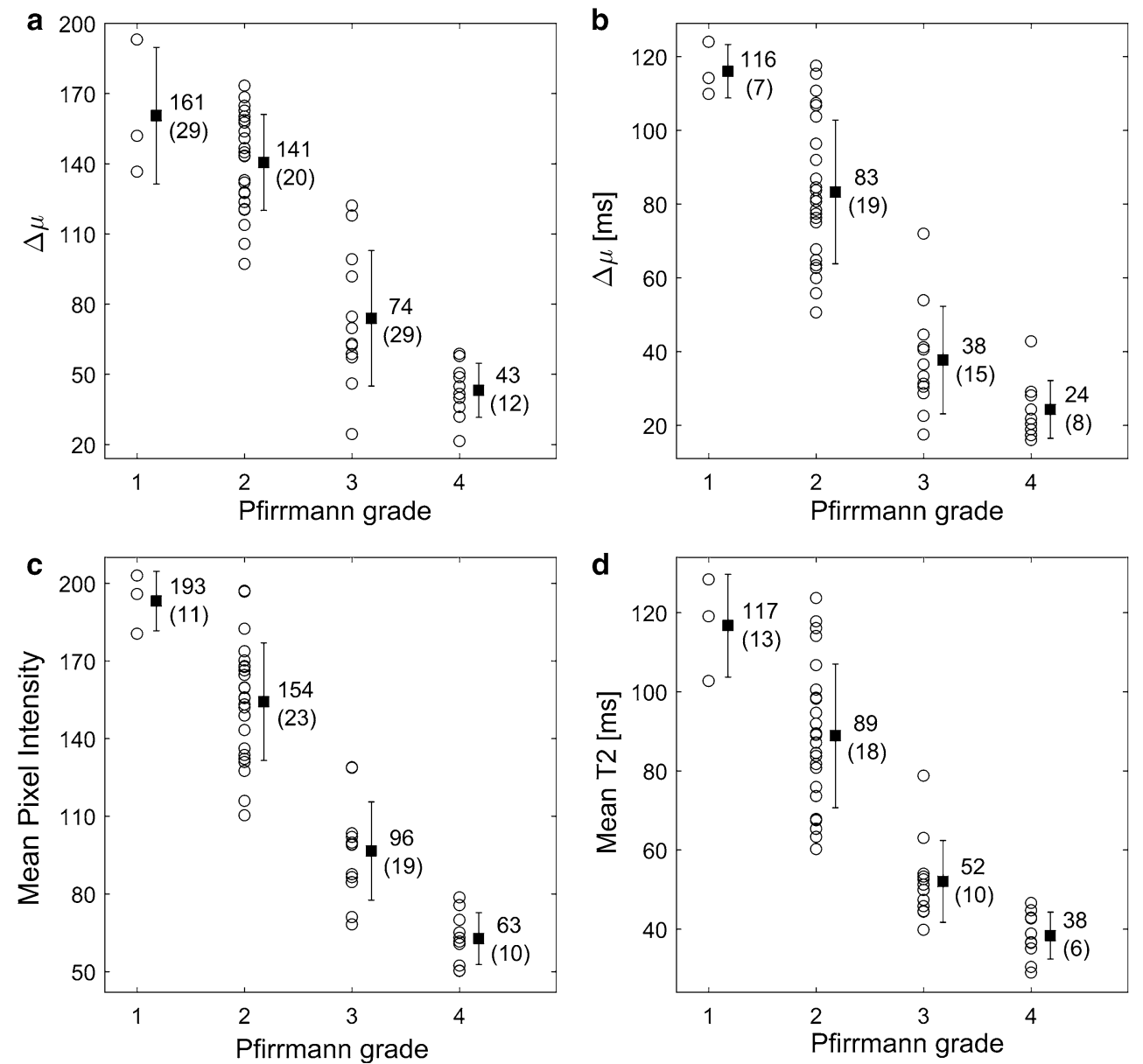

\section{Discussion}

In the pursuit of finding a tool for objective and continuous classification of IVD degeneration, this study investigated IVD grey scale distributions, i.e., histograms. It was shown that the decoding of the IVD heterogeneity with histogram analysis is feasible not only for T2-mapping, but also for conventional $\mathrm{T} 2 \mathrm{~W}$-imaging. This is advantageous as conventional T2W MR images, compared to T2-maps, are frequently used in a clinical setting. Moreover, they offer reduced acquisition time while still maintaining the ability to convert qualitative data into quantitative data. It should be noted that $\mathrm{T} 2 \mathrm{~W}$-imaging is sensitive to the choice of parameters for signal amplification. Consequently, only 
Fig. 4 Example of vastly different histogram topology for discs all graded Pfirrmann 3. Histograms are based on IVD pixel values in a $\mathrm{T} 2 \mathrm{~W}$-images and b T2-maps
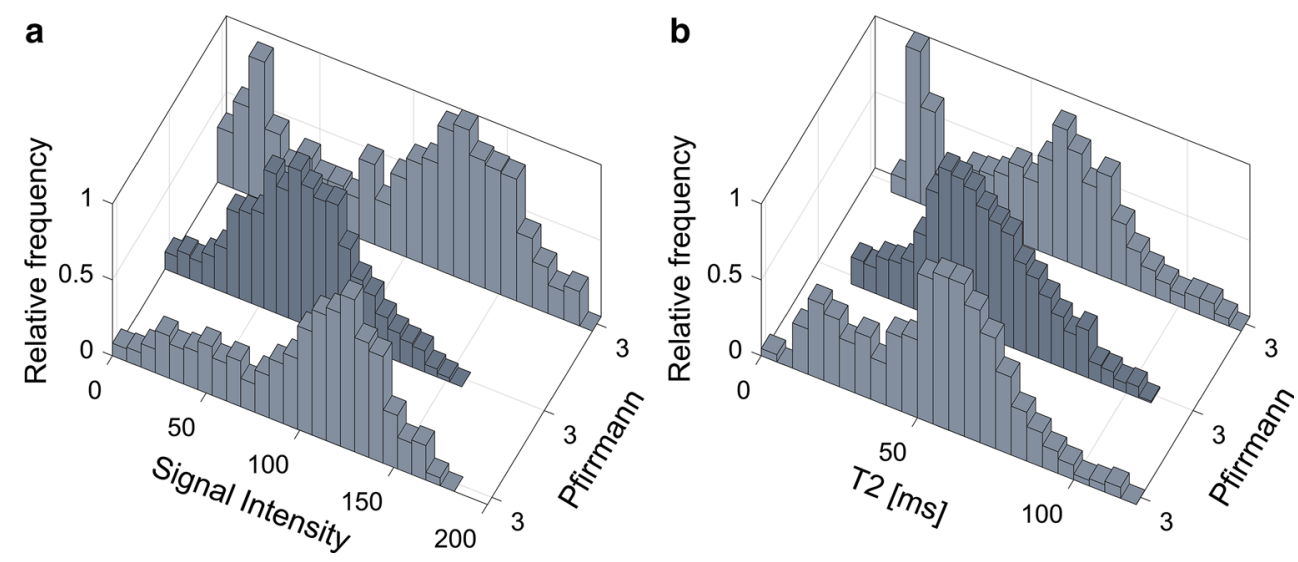
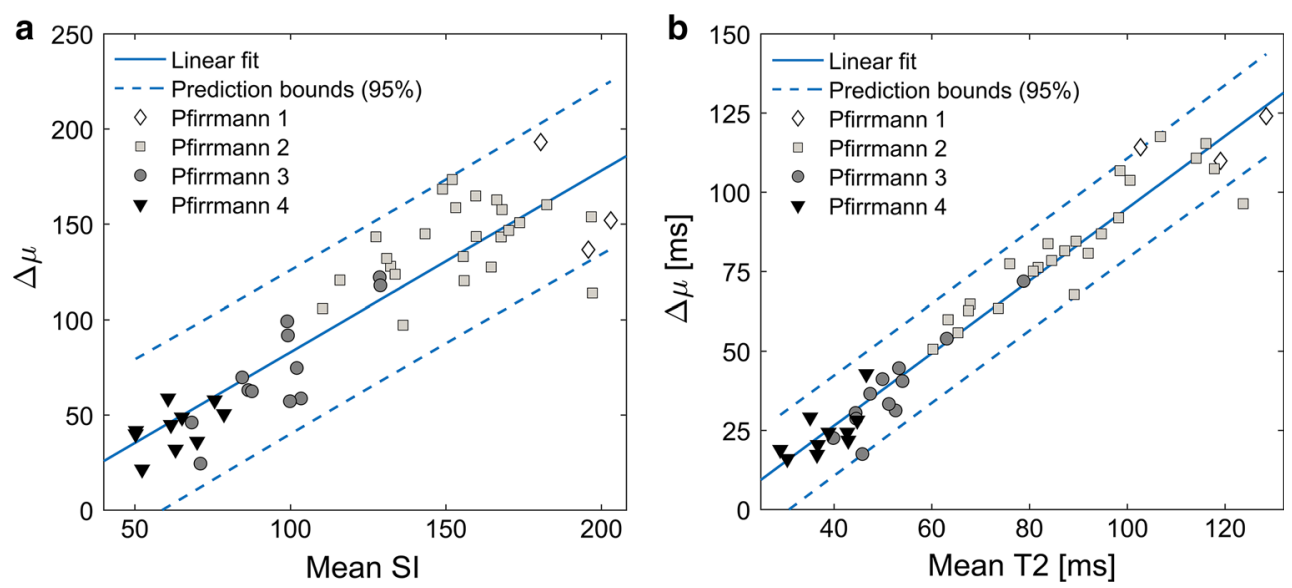

Fig. 5 Gaussian peak separation, $\Delta \mu$, plotted as a function of a mean IVD signal intensity, and $\mathbf{b}$ mean IVD T2 relaxation time

when all $\mathrm{T} 2 \mathrm{~W}$-images are acquired using the same amplification settings in the MRI, as done in this study, the pixel values across all $\mathrm{T} 2 \mathrm{~W}$-images can be compared.

Despite the fact that patients suffering from LBP to some extent have degenerative IVDs, conventional IVD degeneration measurements (Pfirrmann grading) alone cannot identify patients with lumbar pain. In contrast to Pfirrmann grading, which is a coarse and to some extent subjective marker of degeneration as it is based on visual interpretation [9], histogram analysis have the potential to objectively depict differences in the IVDs, not only between different grades, but also within single grades. Histogram analysis may thus produce sensitive IVD histogram features, which can be used for detection of early IVD degeneration, as well as a potential tool to evaluate early therapy response. In addition, IVD degeneration has been shown to correlate to Oswestry Disability Index [18], possibly enabling histogram features to indicate patient disability.

All investigated histogram features presented in this study correlated well with Pfirrmann grade. Compared to other studies, similar T2-values of the IVDs were retrieved [14, 16, 19]. Histogram analysis of IVDs of different Pfirrmann grades generated a wide and continuous range of
$\Delta \mu$ which shows that histogram analysis might be a suitable tool to be used for objective and continuous IVD degeneration classification. Histograms of non-degenerated IVDs based on T2W-images or T2-maps data should display two well separated peaks, one from $\mathrm{AF}$ and one from NP. In the process of IVD degeneration, an increased number of AF fissures are developed that may result in leakage of NP into the AF [20, 21]. Hypothetically, such leakage could be characterized by a region of high signal intensity in the otherwise low signal AF. One example of such high signal intensity in the IVD are High Intensity Zones (HIZ) which represents annular fissures [22]. Hypothetically, due to the common sub-voxel size of annulus fissures, only a minority of these fissures are fully visualized in MR images. Therefore, a detailed analysis and clear visualization of HIZ may be hampered by limited spatial resolution often restricted by the MRI slice thickness width. This might be a reason to the inconclusive research where some studies show HIZ being closely related to pain [23, 24], and other studies do not [25, 26]. Nonetheless, the "invisible" fissures and loss of water and collagen concentration in the NP may be identified by histogram analysis as an increase of intermediate histogram 
values and a decrease in Gaussian peak separation, $\Delta \mu$, reflecting the tissue mixture in the voxel volume.

Histogram analysis revealed that histograms of some IVD grey scale distributions within the same Pfirrmann group exhibit vastly different histogram topology. One hypothesis to this phenomenon is that some IVDs may have a wide dorsal annular rift, visible as a clear HIZ, while other IVDs may have multiple smaller fissures generating more intermediate voxel values. Hence, histogram analysis may possibly generate objective features that are able to indicate differences in IVDs that macroscopically seem comparable, but at disc level differs between symptomatic and asymptomatic IVDs. Although fissures and HIZ have been associated with an ingrowth of nerve endings and granulation tissue, which is a prerequisite to cause pain [15], it is today not established how the distribution of fissures are related to LBP. With the use of quantitative IVD histogram features, extended investigations of the development of fissures within both clinical settings as well as in research can be performed.

This study would have benefitted from including more individuals with IVDs of Pfirmann grade 1 and 5, to statistically determine correlation between histogram features and IVD degeneration in terms of Pfirrmann grade. However, as IVDs of Pfirrmann grade 1 are non-degenerated and IVDs of Pfirrmann grade 5 are extensively degenerated and almost fused, these groups are of less interest regarding interventional measures. Another limitation of this study is that the IVD evaluation covered only a mid-sagittal IVD width of about one centimetre. An evaluation covering a larger IVD volume, without increasing the image slice thickness, could potentially more accurately describe the regional heterogeneity also in lateral regions. It is known that the spine is a dynamic structure which is influenced by axial load or recumbency [27, 28]. As all imaging was done with the patients in a supine position without axial load, the IVD morphological state might have deviated from upright standing conditions. Future studies are warranted to examine the loading effect.

Quantification of unique regional patterns in MR images, such as histogram patterns, is emerging for phenotyping of different tissues. By integrating histogram analysis with automatic techniques for image analysis, e.g. machine learning [29], image data can be effectively extracted in a more clinically feasible way.

Development of a fully automatic segmentation algorithm should be considered as it could reduce variations in the location and size of the ROI and ultimately reduce the spread of calculated data. As only patients with chronic LBP were studied, it is currently unknown if IVD histogram analysis between an asymptomatic and symptomatic group would differ. Studies in which IVD histogram features of asymptomatic and symptomatic individuals are compared are warranted. Such studies might indicate whether histogram features can improve patient management and indicate LBP.

\section{Conclusion}

This study shows that decoding of the IVD heterogeneity with histogram analysis is feasible not only for T2-mapping, but also for conventional T2W-imaging. Histogram features correlated well with IVD degeneration, determined with Pfirrmann grading, suggesting that IVD histogram analysis is a suitable tool for objective and continuous IVD degeneration classification. Moreover, as histogram analysis reveals the IVD heterogeneity, it may be a clinical tool for characterization of regional degeneration effects on IVD structures.

Acknowledgements The authors acknowledge the financial support from C4I center, Sahlgrenska University Hospital and the Innovation Fund, The region of West Sweden.

\section{Compliance with ethical standards}

Conflict of interest Authors declare that they have no conflict of interest.

Ethical approval All procedures performed in studies involving human participants were in accordance with the ethical standards of the institutional and/or national research committee and with the 1964 Helsinki declaration and its later amendments or comparable ethical standards.

Informed consent Oral and written informed consent was obtained from all individual participants included in the study.

Open Access This article is distributed under the terms of the Creative Commons Attribution 4.0 International License (http://crea tivecommons.org/licenses/by/4.0/), which permits unrestricted use, distribution, and reproduction in any medium, provided you give appropriate credit to the original author(s) and the source, provide a link to the Creative Commons license, and indicate if changes were made.

\section{References}

1. Andersson GB (1999) Epidemiological features of chronic lowback pain. Lancet (London, England) 354(9178):581-585. doi:10.1016/s0140-6736(99)01312-4

2. Modic MT, Ross JS (2007) Lumbar degenerative disk disease. Radiology 245(1):43-61. doi:10.1148/radiol.2451051706

3. Hansson EK, Hansson TH (2005) The costs for persons sicklisted more than one month because of low back or neck problems. A two-year prospective study of Swedish patients. Eur Spine J Off Publ Eur Spine Soc Eur Spinal Deform Soc Eur Sect Cerv Spine Res Soc 14(4):337-345. doi:10.1007/s00586-0040731-3

4. Hoy D, March L, Brooks P, Blyth F, Woolf A, Bain C, Williams G, Smith E, Vos T, Barendregt J, Murray C, Burstein R, 
Buchbinder R (2014) The global burden of low back pain: estimates from the Global Burden of Disease 2010 study. Ann Rheum Dis 73(6):968-974. doi:10.1136/annrheumdis-2013204428

5. Luoma K, Riihimäki H, Luukkonen R, Raininko R, ViikariJuntura E, Lamminen A (2000) Low back pain in relation to lumbar disc degeneration. Spine 25(4):487-492

6. Salminen JJ, Erkintalo MO, Pentti J, Oksanen A, Kormano MJ (1999) Recurrent low back pain and early disc degeneration in the young. Spine 24(13):1316-1321

7. Hancock M, Maher C, Macaskill P, Latimer J, Kos W, Pik J (2012) MRI findings are more common in selected patients with acute low back pain than controls? Eur Spine J Off Publ Eur Spine Soc Eur Spinal Deform Soc Eur Sect Cerv Spine Res Soc 21(2):240-246. doi:10.1007/s00586-011-1955-7

8. Jarvik JG, Deyo RA (2002) Diagnostic evaluation of low back pain with emphasis on imaging. Ann Intern Med 137(7):586-597

9. Pfirrmann CW, Metzdorf A, Zanetti M, Hodler J, Boos N (2001) Magnetic resonance classification of lumbar intervertebral disc degeneration. Spine 26(17):1873-1878

10. Jenkins JPR, Hickey DS, Zhu XP, Machin M, Isherwood I (1985) MR imaging of the intervertebral disc: a quantitative study. Br J Radiol 58(692):705-709. doi:10.1259/0007-1285-58-692-705

11. Auerbach JD, Johannessen W, Borthakur A, Wheaton AJ, Dolinskas CA, Balderston RA, Reddy R, Elliott DM (2006) In vivo quantification of human lumbar disc degeneration using T1 $\rho$-weighted magnetic resonance imaging. Eur Spine J 15(3):338-344. doi:10.1007/s00586-006-0083-2

12. Stelzeneder D, Welsch GH, Kovács BK, Goed S, PaternostroSluga T, Vlychou M, Friedrich K, Mamisch TC, Trattnig S (2012) Quantitative T2 evaluation at 3.0 T compared to morphological grading of the lumbar intervertebral disc: a standardized evaluation approach in patients with low back pain. Eur $\mathbf{J}$ Radiol 81(2):324-330. doi:10.1016/j.ejrad.2010.12.093

13. Michopoulou S, Costaridou L, Vlychou M, Speller R, ToddPokropek A (2011) Texture-based quantification of lumbar intervertebral disc degeneration from conventional T2-weighted MRI. Acta Radiol (Stockholm, Sweden: 1987) 52(1):91-98. doi:10.1258/ar.2010.100166

14. Watanabe A, Benneker LM, Boesch C, Watanabe T, Obata T, Anderson SE (2007) Classification of intervertebral disk degeneration with axial T2 mapping. AJR Am J Roentgenol 189(4):936-942. doi:10.2214/ajr.07.2142

15. Brayda-Bruno M, Tibiletti M, Ito K, Fairbank J, Galbusera F, Zerbi A, Roberts S, Wachtel E, Merkher Y, Sivan SS (2014) Advances in the diagnosis of degenerated lumbar discs and their possible clinical application. Eur Spine J 23(Suppl 3):S315-S323. doi:10.1007/s00586-013-2960-9

16. Marinelli NL, Haughton VM, Anderson PA (2010) T2 relaxation times correlated with stage of lumbar intervertebral disk degeneration and patient age. AJNR Am J Neuroradiol 31(7):1278-1282. doi:10.3174/ajnr.A2080

17. Welsch GH, Trattnig S, Paternostro-Sluga T, Bohndorf K, Goed S, Stelzeneder D, Mamisch TC (2011) Parametric T2 and T2* mapping techniques to visualize intervertebral disc degeneration in patients with low back pain: initial results on the clinical use of
3.0 Tesla MRI. Skelet Radiol 40(5):543-551. doi:10.1007/ s00256-010-1036-8

18. Middendorp M, Vogl TJ, Kollias K, Kafchitsas K, Khan MF, Maataoui A (2016) Association between intervertebral disc degeneration and the Oswestry Disability Index. J Back Musculoskelet Rehabil. doi:10.3233/bmr-150516

19. Kerttula L, Kurunlahti M, Jauhiainen J, Koivula A, Oikarinen J, Tervonen O (2001) Apparent diffusion coefficients and T2 relaxation time measurements to evaluate disc degeneration: a quantitative MR study of young patients with previous vertebral fracture. Acta Radiol 42(6):585-591. doi:10.1080/ 028418501127347241

20. McCarron RF, Wimpee MW, Hudkins PG, Laros GS (1987) The inflammatory effect of nucleus pulposus: a possible element in the pathogenesis of low-back pain. Spine 12(8):760-764

21. Sugiura K, Tonogai I, Matsuura T, Higashino K, Sakai T, Suzue N, Hamada D, Goto T, Takata Y, Nishisho T, Goda Y, Sato R, Kondo K, Tezuka F, Mineta K, Takeuchi M, Takahashi M, Egawa H, Sairyo K (2014) Discoscopic findings of high signal intensity zones on magnetic resonance imaging of lumbar intervertebral discs. Case Rep Orthop 2014:5. doi:10.1155/2014/ 245952

22. Aprill C, Bogduk N (1992) High-intensity zone: a diagnostic sign of painful lumbar disc on magnetic resonance imaging. $\mathrm{Br} \mathrm{J}$ Radiol 65(773):361-369. doi:10.1259/0007-1285-65-773-361

23. Peng B, Hou S, Wu W, Zhang C, Yang Y (2006) The pathogenesis and clinical significance of a high-intensity zone (HIZ) of lumbar intervertebral disc on MR imaging in the patient with discogenic low back pain. Eur Spine J 15(5):583-587. doi:10. 1007/s00586-005-0892-8

24. Schellhas KP, Pollei SR, Gundry CR, Heithoff KB (1996) Lumbar disc high-intensity zone: correlation of magnetic resonance imaging and discography. Spine 21(1):79-86

25. Pande KC, Khurjekar K, Kanikdaley V (2009) Correlation of low back pain to a high-intensity zone of the lumbar disc in Indian patients. J Orthop Surg (Hong Kong) 17(2):190-193

26. Hebelka H, Hansson T (2013) HIZ's relation to axial load and low back pain: investigated with axial loaded MRI and pressure controlled discography. Eur Spine J 22(4):734-739. doi:10.1007/ s00586-012-2501-y

27. Nilsson M, Lagerstrand K, Kasperska I, Brisby H, Hebelka H (2016) Axial loading during MRI influences T2-mapping values of lumbar discs: a feasibility study on patients with low back pain. Eur Spine J 25(9):2856-2863. doi:10.1007/s00586-0164670-6

28. Belavy DL, Quittner M, Ling Y, Connell D, Rantalainen T (2017) Cervical and thoracic intervertebral disc hydration increases with recumbency: a study in 101 healthy volunteers. Spine J. doi:10. 1016/j.spinee.2017.06.006

29. Jamaludin A, Lootus M, Kadir T, Zisserman A, Urban J, Battie MC, Fairbank J, McCall I (2017) ISSLS PRIZE IN BIOENGINEERING SCIENCE 2017: automation of reading of radiological features from magnetic resonance images (MRIs) of the lumbar spine without human intervention is comparable with an expert radiologist. Eur Spine J 26(5):1374-1383. doi:10.1007/ s00586-017-4956-3 